\title{
Nursing Home Revenue Source and Information Availability During the Emergency Department Evaluation of Nursing Home Residents
}

\author{
Timothy F. Platts-Mills, MD, Kevin Biese, MD, MAT, Michael LaMantia, MD, MPH, Zeke \\ Zamora, MD, Laura N. Patel, MD, Brenda McCall, RN, Fortune Egbulefu, Jan Busby- \\ Whitehead, MD, Charles B. Cairns, MD, and John S. Kizer, MD \\ Department of Emergency Medicine, University of North Carolina, Chapel Hill (Platts-Mills, Biese, \\ Cairns). Division of Geriatrics, Department of Medicine, University of North Carolina, Chapel Hill \\ (LaMantia, Zamora, Patel, McCall, Busby-Whitehead, Kizer). School of Medicine, University of \\ North Carolina, Chapel Hill (Egbulefu)
}

\begin{abstract}
OBJECTIVES—Lack of access to medical information for nursing home residents during Emergency Department (ED) evaluation is a barrier to quality care. We hypothesized that the quantity of information available in the ED differs based on the funding source of the resident's nursing home.

DESIGN—Cross-sectional observational study.

SETTING-Single academic ED.

PARTICIPANTS-128 skilled nursing facility (SNF) residents age 65 or older from 12 SNFs.

MEASUREMENTS-Emergency physicians documented knowledge of 9 essential information items. SNFs were categorized as accepting or not accepting Medicaid.

RESULTS-Questionnaires were completed for 128 patients, of whom 95 (74\%) were from one of 8 Medicaid-funded SNFs and 33 (26\%) were from one of 4 SNFs not accepting Medicaid. Patients from SNFs accepting Medicaid were younger (79 vs. 87, p<.001) and less frequently white (62\% vs. $97 \%, \mathrm{p}<.001)$. The mean number of 9 possible information items available was lower for patients from SNFs that accept Medicaid (7.13 vs. 8.15, $\mathrm{p}<.001$ ). Emergency providers also reported lower satisfaction regarding access to information for residents from SNFs that accept Medicaid $(\mathrm{p}<.05)$. The association between residence in a SNF that accepts Medicaid and lower ED information scores remained after linear regression with clustering by SNF controlling
\end{abstract}

(C) 2010 American Medical Directors Association. Published by Elsevier Inc. All rights reserved.

Corresponding Author: Timothy F. Platts-Mills, M.D., Department of Emergency Medicine, University of North Carolina Chapel Hill, 170 Manning Drive, CB \#7594, Chapel Hill, NC 27599-7594, tplattsm@ @ed.unc.edu, Phone: 559-240-6073, Fax: 919-966-3049. Alt. Corresponding Author: Kevin Biese, MD, MAT; kbiese@med.unc.edu.

Offprints: Offprints will not be available from the author.

Publisher's Disclaimer: This is a PDF file of an unedited manuscript that has been accepted for publication. As a service to our customers we are providing this early version of the manuscript. The manuscript will undergo copyediting, typesetting, and review of the resulting proof before it is published in its final citable form. Please note that during the production process errors may be discovered which could affect the content, and all legal disclaimers that apply to the journal pertain.

Presentations: Presented as a poster at the Society for Academic Emergency Medicine National Meeting in Phoenix, Arizona, June 2010.

Conflict of Interest: None of the authors have any conflicts of interest with the material reported here. Specifically, no author holds or has received employment, grants or funds, honoraria, speaker forum, consultant, stocks, royalties, expert testimony, board membership, patents or personal relationships which constitutes a conflict of interest with the work presented here. 
for age, gender, and race. The most common source of information for residents from both types of SNFs was transfer papers from the SNF.

CONCLUSION-Less information is available to ED providers for patients from SNFs that accept Medicaid than for residents from SNFs that do not accept Medicaid. Further study is needed to examine this information gap.

\section{INTRODUCTION}

Emergency Department (ED) visits for elderly adults have increased in the past 10 years. 1 Elderly ED patients tend to have more complex illnesses than younger patients, with higher triage scores, longer ED length of stays, higher rates of hospital and intensive care unit admission, and higher rates of return visits.2 Elderly adults who reside in skilled nursing facilities (SNFs) use the ED at particularly high rates, with an estimate of visit rates exceeding one visit per patient per year.3 Essential patient information for elderly SNF residents is often unavailable to ED providers. $4^{-7}$ In particular, the patient's reason for visit, baseline mental and physical function, and advance directives are commonly not available.6, 7 The absence of readily available medical information has been identified by emergency medicine physicians as a common problem and a barrier to providing appropriate and timely care for elderly ED patients. $8^{-} 10$ Thus, a lack of health information in this population may both impede the timely care of acutely ill individuals and also lead to the overuse of diagnostic tests and unnecessary hospital admissions. The magnitude of this problem is likely to increase over the next two decades as the number of older adults in nursing homes grows due to aging of the baby boom population. Current estimates indicate that the population of nursing home residents will increase over this time period despite reductions in the prevalence of disability.11

Socioeconomic status is a powerful determinant of access to care, quality of care, and health care outcomes in the United States.12-16 More specifically, nursing home revenue source and reimbursement rates have previously been linked to both staffing levels and quality of care.17, 18 Although several studies have reported on the deficiencies in patient information available to ED providers for patients from SNFs, no studies have examined the relationship between nursing home revenue source and the amount of information available to ED providers. The objective of this study is to examine the relationship between nursing home revenue source and the quantity of patient information available at the time of ED evaluation.

\section{METHODS}

\section{Setting and Participants}

This study was conducted at a single Level I trauma center ED with an emergency medicine residency program providing service to a large and socioeconomically diverse elderly population between January and July of 2009. The ED has an annual census of approximately 65,000 patients. Of these, approximately 8,600 (13\%) patients each year are age 65 or older and approximately 700 are both age 65 or older and are residents of a SNF. The sending SNFs are located in suburban and rural areas within the county where the ED is located and three surrounding counties. The study site ED is the primary hospital for these SNFs. We enrolled consecutive patients age 65 or older who arrived at the ED from a SNF between 8 a.m. and 5 p.m. Data collection was limited to day time hours to allow the geriatric research nurse responsible for data collection to ensure the completion of questionnaires by emergency medicine residents. For inclusion, patients had to be sent to the ED directly from their SNF. Patients residing in SNFs but sent to the ED from another site (e.g. physician office or dialysis center) were not included. Skilled nursing facilities were 
categorized into two groups based on their acceptance or non-acceptance of Medicaidfunded patients as defined on the Medicare.gov Nursing Home Compare website.19

All SNFs that sent study patients to the ED were contacted by a dedicated, trained geriatric research nurse during the study period to confirm the Nursing Home Compare categorizations and to obtain further information about the resources available in these facilities. Admissions coordinators from each SNF were interviewed by the study nurse to obtain information about the number of residents in the nursing home, payer source for these residents, the use of electronic medical records, and the ratios of residents to registered nurses and nursing assistants. All phone calls to SNFs were made during a two week period at the end of the study. Nursing staff turnover rates were provided by the University of North Carolina's Institute on Aging as part of an ongoing study of geriatric provider supply. The hospital's Institutional Review Board reviewed and approved this study with a waiver of informed consent.

\section{Data Collection}

Questionnaires were completed by treating emergency medicine residents at the end of the patient's ED care. All ED residents were trained on how to complete the form before the study began and received regular reminders throughout the study. Additionally, the form contained explicit instructions to the treating physician to assist them in determining the presence of absence of specific information items at any time during the patient's ED evaluation. Consistent with recent prior studies assessing the availability of ED information for SNFs patients during transitions, a dichotomous outcome for each information item was used.20 Residents were instructed to mark an information item as present if they were able to obtain some information for that item. For example, if the treating physician obtained a list of medications, that information was marked as present even if the physician was not sure if the medication list was complete. Treating physicians were also instructed to accept all possible sources of information in determining the presence or absence of each information item and record the source of the study questionnaire. For each of the 9 items that were available, residents chose from one of 7 possible sources of information: the patient, emergency medical service providers, family, paper work sent from the SNF, conversations with SNF representatives via phone, and electronic medical records.

Residents were aware that data was being collected on the availability of information to ED providers for SNF patients but were not informed about the investigator's interest in the association between information availability and the SNF's funding source. Additional demographic information was abstracted from the medical record by a dedicated geriatric research nurse who has formal training in chart abstraction methodology using a standardized electronic data template (Microsoft Excel 2007, Microsoft Corp. Redmond, WA).

\section{Outcome Measures}

The primary outcome measure was the quantity of information available to ED providers. Whether information was present was assessed by the ED provider and recorded on a questionnaire. Consistent with recent studies examining information transfer for SNF patients, we used 9 items to assess the availability of information for each patient.6, 20-22 The 9 information items were: 1) reason for visit, 2) past medical history, 3) medications, 4) allergies, 5) baseline mental status, 6) baseline ambulatory status, 7) primary provider name, 8) healthcare power of attorney name, 9) advance directives. Each information item was given a score of 1 if present or 0 if not present, and the sum of the 9 information items was used to obtain a summary score of information availability. 
Secondary outcomes included the availability of each of the 9 individual outcome measures as well as the resident's overall satisfaction with the information available for the patient. Residents scored their satisfaction with the availability of information on a scale from one to four, with: $1=$ completely unsatisfied; $2=$ mostly unsatisfied; $3=$ mostly satisfied; and $4=$ completely satisfied.

\section{Statistical Analysis}

The t-test for independent samples was used to compare the mean quantity of information available for patients from each of the two groups of nursing homes. This analysis was repeated using the Wilcoxon rank-sum test to assess for the effect of a non-normal distribution of information scores. The t-test was also used to compare the size, staffing and turnover rates of SNFs that accept and do not accept Medicaid. Fisher's exact test was used to compare the proportions of patients with individual information items available from each nursing home and compare the availability of electronic medical records for the two groups of SNFs. Results for 2-sided comparisons are reported for all tests. Linear regression analysis with clustering around SNF and controlling for patient age, gender, and race was used to determine the association between nursing home funding source and the quantity of information available. For this analysis, we used forced entry of the listed demographic variables. Statistical analyses were performed using PASW (version 17.0; SPSS Inc., Chicago, IL) and Stata (version 10.0; StataCorp, College Station, TX). The contribution of each of the 7 possible sources of information is reported in two ways. First, we provide the number of patients who had at least one of their 9 information items primarily from this source. Second, we report the mean number of information items for which the source was the primary means by which the emergency physician obtained information about the patient.

Prior to initiation of the study, a minimum sample size of 63 patients in each group was determined to be sufficient to provide $80 \%$ power $(a=.05)$ to detect a 1 point increase in the quantity of information available for patients from each type of nursing home assuming a mean information score of 8 and a standard deviation of 2 . This calculation assumed equal numbers of patients from each of the two categories of nursing homes. Based on this, we planned to collect data on 126 patients.

\section{RESULTS}

Physicians completed questionnaires for 128 patients. Study patients $(n=128)$ were similar to patients who came to the ED during the months of the study but after hours or on weekends $(\mathrm{n}=362)$ in regard to age ( 82.6 vs. 83.5$)$, race $(76.2 \%$ vs. $75.3 \%$ white; $20.3 \%$ vs. $22.0 \%$ black), and gender (67.1\% vs. $68.6 \%$ female). Of the 128 study patients, 95 (74\%) were from eight different SNFs that accept Medicaid and 33 (26\%) were from four different SNFs that do not accept Medicaid. All study patients had Medicare insurance. Of the 95 patients from SNFs that accept Medicaid 26 (27.4\%) had Medicaid insurance. None of the patients from SNFs that do not accept Medicaid had Medicaid insurance. Patients from SNFs that accept Medicaid were younger (79 vs. 87, p<.001) and less frequently white (65\% vs. $97 \%$, $\mathrm{p}<.01$ ) than patients from SNFs that do not accept Medicaid.

Skilled nursing facilities that accept Medicaid ( $\mathrm{n}=8)$ reported more total beds (122 vs. 42, $\mathrm{p}=.005$ ), were closer to capacity ( $95 \%$ vs. $87 \%, \mathrm{p}<.05)$, and had higher annual nursing staff turnover rates $(117 \%$ vs. $33 \%, \mathrm{p}<.05)$ than did SNFs that do not accept Medicaid (Table 1). Only one of the eight referring SNFs that accept Medicaid reported using electronic medical records whereas all four of the SNFs that do not accept Medicaid reported using electronic medical records $(\mathrm{p}<.01)$. 
The mean quantity of information available for patients from SNFs that accept Medicaid was less than for patients from SNFs that do not accept Medicaid (7.13 vs. 8.15, p<.001). Non-parametric testing also revealed a significant difference with medians of 8 vs. 9 , respectively $(p<0.05)$. For each of the 9 information items, the percentage of patients for whom the information was available was lower for patients from SNFs accepting Medicaid than for patients not accepting Medicaid (Table 2). These differences were significant for information about medications ( $\mathrm{p}<.05)$ and advance directives ( $\mathrm{p}<.01)$. Mean ED provider scores for satisfaction with the availability of information were lower for patients from SNFs that accept Medicaid than for patients from SNFs that do not accept Medicaid (2.8 vs. 3.2, $\mathrm{p}<.05)$.

In multivariable linear regression with clustering by SNF, residence in a SNF that does not accept Medicaid ( $<<.005)$, male gender $(p<.005)$, and older age of the patient $(p<.05)$ were associated with increased information availability in the ED. Patient race $(\mathrm{p}=.93)$ was not associated with the availability of information.

The dominant source of information for patients from both types of SNFs was transfer papers sent with the patient from the nursing home (Table 3). Electronic health records were a source of at least one information item for almost half (48\%) of patients from SNFs accepting Medicaid vs. a third (33\%) of patients from SNFs not accepting Medicaid. The contributions of the patient and of family members to information availability were similar for patients from the two types of SNFs.

\section{DISCUSSION}

In this study of elderly patients presenting to the ED from a diverse group of nursing homes, we found the emergency physicians reported less availability of important medical information for patients from nursing homes that accept Medicaid funding. Specific items for which information was less frequently available in patients from SNFs that accept Medicaid were medications and advance directives. Additionally, ED providers were less satisfied on average with the information available for patients from SNFs that accept Medicaid. The difference in information availability for the two types of SNFs persisted after adjusting for patient age, gender, race and SNF. These findings suggest that the lack of information availability during transfer of residents from SNFs to EDs may be a bigger problem for patients from SNFs that accept Medicaid.

The two particular information items, current medications and advance directive status, which were less often present for patients from SNFs that accept Medicaid are clinically important. Medication effects are a common cause of ED visits and hospital admissions for this population, 23,24 and the presence of certain medications is a contraindication to key therapeutic interventions for acute severe illness (e.g. coumadin use is a contraindication to the use of thrombolytics for acute ischemic stroke). Additionally, gaps in medication information have been linked to drug related problems during hospitalization.25 Thus, we feel that the optimal evaluation and treatment of elderly nursing home patients is impossible without a medication list. Similarly, a number of therapeutic decisions may be affected by a patient's advance directives. In the absence of this information, emergency physicians generally presume that full care is desired, because the alternative may result in a wrongful death.7 The absence of information about advance directives and more generally the care preferences of nursing home residents may expose patients to care that they do not desire or result in unwarranted resource utilization.26, 27

We used whether or not the SNF accepts Medicaid to categorize the SNFs into two groups because this information is publicly available, allows for a widely reproducible approach, 
and appears to accurately distinguish between two different types of SNFs in regard to the SNFs' resources. Additionally, Medicaid is the dominant purchaser of nursing home services in the United States and is probably the most important single predictor of the resources available for those nursing homes which accept Medicaid payments. 18 The differences in size, nursing staff turnover rates, and the use of electronic medical records for the two types of SNFs (Table 1) support this method of categorization. We chose to use the $\mathrm{SNF}$ as the unit of measure for socioeconomic status, not the patient, because it is our impression that the information transferred from SNFs to EDs typically reflects the policies and activities of the SNF rather than financial status of the individual patient.

Our study identifies an association between a socioeconomic categorization of SNFs and the availability of information in the ED. There are many possible explanations for this association. In our community, the SNFs that accept Medicaid have fewer financial resources than those that do not accept Medicaid. Additionally, SNFs that accept Medicaid are larger, operate closer to capacity, are less likely to use electronic medical records, and have higher nursing staff turnover rates. Each of these factors may impact the quantity of information transferred during an ED visit. Other factors such as duration of patient stay at an SNF, co-morbidities, cognitive impairment, and being accompanied by family members or care providers during ED visits may also influence the availability of information in the ED and may vary between SNFs that do and do not accept Medicaid. Finally, emergency medical services and ED providers may take different attitudes towards patients from each type of SNF, and these differences in attitude might affect the effort made in obtaining information. Because of the strong correlation between these variables, our data does not allow us to differentiate among SNF payer source, SNF size, and nursing stuff turnover rate as the primary determinant of the quantity of ED information. A larger study with greater variability in SNF sizes and turnover rates will be needed in order to isolate the contribution of these various factors to the outcome of interest.

The information we collected on the source of information suggests that the increased availability of information is not simply the result of increased use of a single information source. Rather, for patients from SNFs that accept Medicaid there was a tendency towards having fewer information items from all sources (bottom 2 rows, Table 3) with the exception of family members. Further, electronic health records made only a small contribution to the total information available even for patients from SNFs which do not accept Medicaid, all of which use electronic health records.

It is important to understand the reason for the association identified in this study between SNF socioeconomic status and ED information because such an understanding will likely serve to direct interventions to improve access to information for all SNF patients. A number of the factors associated with SNFs that accept Medicaid may be amenable to policy solutions such as creating incentives to reduce nursing staff turnover rates or increase use of electronic medical records. However, our study was not powered to examine the relative contribution of various sources of information to information availability for patients from the two types of SNFs and further study is need to inform any such policy recommendations.

The difference in information availability between SNFs that do and do not accept Medicaid was present after controlling for age, gender, and race and clustering around individual SNFs. We believe that the difference we have identified represents a difference between two categories of SNFs and is not simply the reflection of variations amongst individual SNFs within the two groups. In multivariate regression, older patient age was associated with increased information. There are several plausible explanations for this association. Because of the association between socioeconomic status and life expectancy,28 advanced age may serve as a surrogate marker for wealth. Alternatively, older SNF residents may have 
different co-morbidities, family support, and degrees of cognitive impairment than younger patients, and these factors may influence the availability of information in the ED.

This study has several limitations. The study was conducted at a single ED with study patients coming from twelve SNFs. It is possible that other EDs and SNFs have different mechanisms and resources to support information transfer. Our study design provided us with a measure of the quantity of information available. While we did not directly address information quality, emergency providers reported higher satisfaction with the availability of information for patients from SNFs that do not accept Medicaid, which might be interpreted as a subjective measure of quality. Further, we asked emergency medicine residents to report on whether the information was available at any time during the ED evaluation and did not qualify this with a measure of when it became available. A more comprehensive assessment of the availability of information would include measures of accuracy and timeliness. We collected data during daytime hours when a geriatric research nurse was available to prompt physicians to initiate the questionnaire and ensure its completeness. Demographic characteristics of study patients were similar to characteristics of SNFs patients who presented to the EDs on nights and weekends, but information availability may be different during off-hours. Patients with frequent ED visits and hospitalizations probably have more information available in hospital medical records than those who rarely visit the ED. Our comparison of sources of information for the two types of SNFs (Table 3) suggests that electronic health records alone probably did not account for the differences in the quantity of information available for patients from the two types of SNFs.

Finally, we did not examine the quality of ED or hospital care or patient outcomes. We and others believe that the availability of medical information in the ED is a determinant of the quality of care, particularly for elderly SNF patients with multiple medical problems and a high frequency of cognitive impairment.9, $20^{-} 22$ A larger study which included outcome measures such as ED length of stay, test ordering, and compliance with quality of care measures might demonstrate a relationship between the availability of information and the quality of care for this population. Because there are many determinants of the quality of care and because in this population higher quality care in some cases means fewer tests, we believe this relationship might be difficult to demonstrate without a much larger study.

In conclusion, our findings demonstrate that the quantity of information available at the time of ED evaluation is lower for patients from SNFs that accept Medicaid than for patients from SNFs that do not accept Medicaid. Further research is needed to confirm the presence of a gap in information availability during SNF-to-ED transitions for patients from these two types of SNFs, identify the causes for this gap, and develop methods to address it.

\section{Acknowledgments}

Funding Source: Work on this project was supported by an Investments for the Future Grant from the UNC School of Medicine to the Center for Aging and Health. Dr. Platts-Mills is supported by grant 5KL2RR025746-03.

Role of the Sponsor: None.

\section{REFERENCES}

1. Roberts DC, McKay MP, Shaffer A. Increasing rates of emergency department visits for elderly patients in the United States, 1993 to 2003. Ann Emerg Med. 2008; 51(6):769-774. [PubMed: 18069088]

2. Strange GR, Chen EH. Use of emergency departments by elder patients: a five-year follow-up study. Acad Emerg Med. 1998; 5(12):1157-1162. [PubMed: 9864128] 
3. Ackermann RJ, Kemle KA, Vogel RL, et al. Emergency department use by nursing home residents. Ann Emerg Med. 1998; 31(6):749-757. [PubMed: 9624316]

4. Turrell AR, Castleden CM. Improving the emergency medical treatment of older nursing-home residents. Age Ageing. 1999; 28(1):77-82. [PubMed: 10203209]

5. Gaddis GM. Elder care transfer forms. Acad Emerg Med. 2005; 12(2):160-161. [PubMed: 15692139]

6. Jones JS, Dwyer PR, White LJ, et al. Patient transfer from nursing home to emergency department: outcomes and policy implications. Acad Emerg Med. 1997; 4(9):908-915. [PubMed: 9305434]

7. Lahn M, Friedman B, Bijur P, et al. Advance directives in skilled nursing facility residents transferred to emergency departments. Acad Emerg Med. 2001; 8(12):1158-1162. [PubMed: 11733294]

8. Gillespie SM, Gleason LJ, Karuza J, et al. Health care providers' opinions on communication between nursing homes and emergency departments. J Am Med Dir Assoc. 11(3):204-210. [PubMed: 20188318]

9. Coleman EA, Berenson RA. Lost in transition: challenges and opportunities for improving the quality of transitional care. Ann Intern Med. 2004; 141(7):533-536. [PubMed: 15466770]

10. McNamara RM, Rousseau E, Sanders AB. Geriatric emergency medicine: a survey of practicing emergency physicians. Ann Emerg Med. 1992; 21(7):796-801. [PubMed: 1610035]

11. Laditka SB. Modeling lifetime nursing home use under assumptions of better health. J Gerontol B Psychol Sci Soc Sci. 1998; 53(4):S177-S187. [PubMed: 9679519]

12. Gornick ME, Eggers PW, Reilly TW, et al. Effects of race and income on mortality and use of services among Medicare beneficiaries. N Engl J Med. 1996; 335(11):791-799. [PubMed: 8703185]

13. Lantz PM, House JS, Lepkowski JM, et al. Socioeconomic factors, health behaviors, and mortality: results from a nationally representative prospective study of US adults. JAMA. 1998; 279(21): 1703-1708. [PubMed: 9624022]

14. Sorlie PD, Backlund E, Keller JB. US mortality by economic, demographic, and social characteristics: the National Longitudinal Mortality Study. Am J Public Health. 1995; 85(7):949_ 956. [PubMed: 7604919]

15. Brook RH, Kamberg CJ, Lohr KN, et al. Quality of ambulatory care. Epidemiology and comparison by insurance status and income. Med Care. 1990; 28(5):392-433. [PubMed: 2187119]

16. Kahn KL, Pearson ML, Harrison ER, et al. Health care for black and poor hospitalized Medicare patients. JAMA. 1994; 271(15):1169-1174. [PubMed: 8151874]

17. Cohen JW, Spector WD. The effect of Medicaid reimbursement on quality of care in nursing homes. J Health Econ. 1996; 15(1):23-48. [PubMed: 10157427]

18. Grabowski DC. A longitudinal study of Medicaid payment, private-pay price and nursing home quality. Int J Health Care Finance Econ. 2004; 4(1):5-26. [PubMed: 15170962]

19. Medicare.gov Nursing Home Compare. 2010 [cited 2010 August 6th]. Available from: http://www.medicare.gov/NHCompare/Include/DataSection/Questions/ProximitySearch.asp.

20. Hustey FM, Palmer RM. An Internet-Based Communication Network for Information Transfer During Patient Transitions from Skilled Nursing Facility to the Emergency Department. J Am Geriatr Soc.

21. Madden C, Garrett J, Busby-Whitehead J. The interface between nursing homes and emergency departments: a community effort to improve transfer of information. Acad Emerg Med. 1998; 5(11):1123-1126. [PubMed: 9835479]

22. Terrell KM, Brizendine EJ, Bean WF, et al. An extended care facility-to-emergency department transfer form improves communication. Acad Emerg Med. 2005; 12(2):114-118. [PubMed: 15692130]

23. Somers A, Robays H, Vander Stichele R, et al. Contribution of drug related problems to hospital admission in the elderly. J Nutr Health Aging. 14(6):477-482. [PubMed: 20617292]

24. Gallagher PF, Barry PJ, Ryan C, et al. Inappropriate prescribing in an acutely ill population of elderly patients as determined by Beers' Criteria. Age Ageing. 2008; 37(1):96-101. [PubMed: 17933759] 
25. Ong SW, Fernandes OA, Cesta A, et al. Drug-related problems on hospital admission: relationship to medication information transfer. Ann Pharmacother. 2006; 40(3):408-413. [PubMed: 16492794]

26. Terrell KM, Miller DK. Challenges in transitional care between nursing homes and emergency departments. J Am Med Dir Assoc. 2006; 7(8):499-505. [PubMed: 17027627]

27. Murray LM, Laditka SB. Care transitions by older adults from nursing homes to hospitals: implications for long-term care practice, geriatrics education, and research. J Am Med Dir Assoc. 11(4):231-238. [PubMed: 20439041]

28. Stringhini S, Sabia S, Shipley M, et al. Association of socioeconomic position with health behaviors and mortality. JAMA. 303(12):1159-1166. [PubMed: 20332401] 


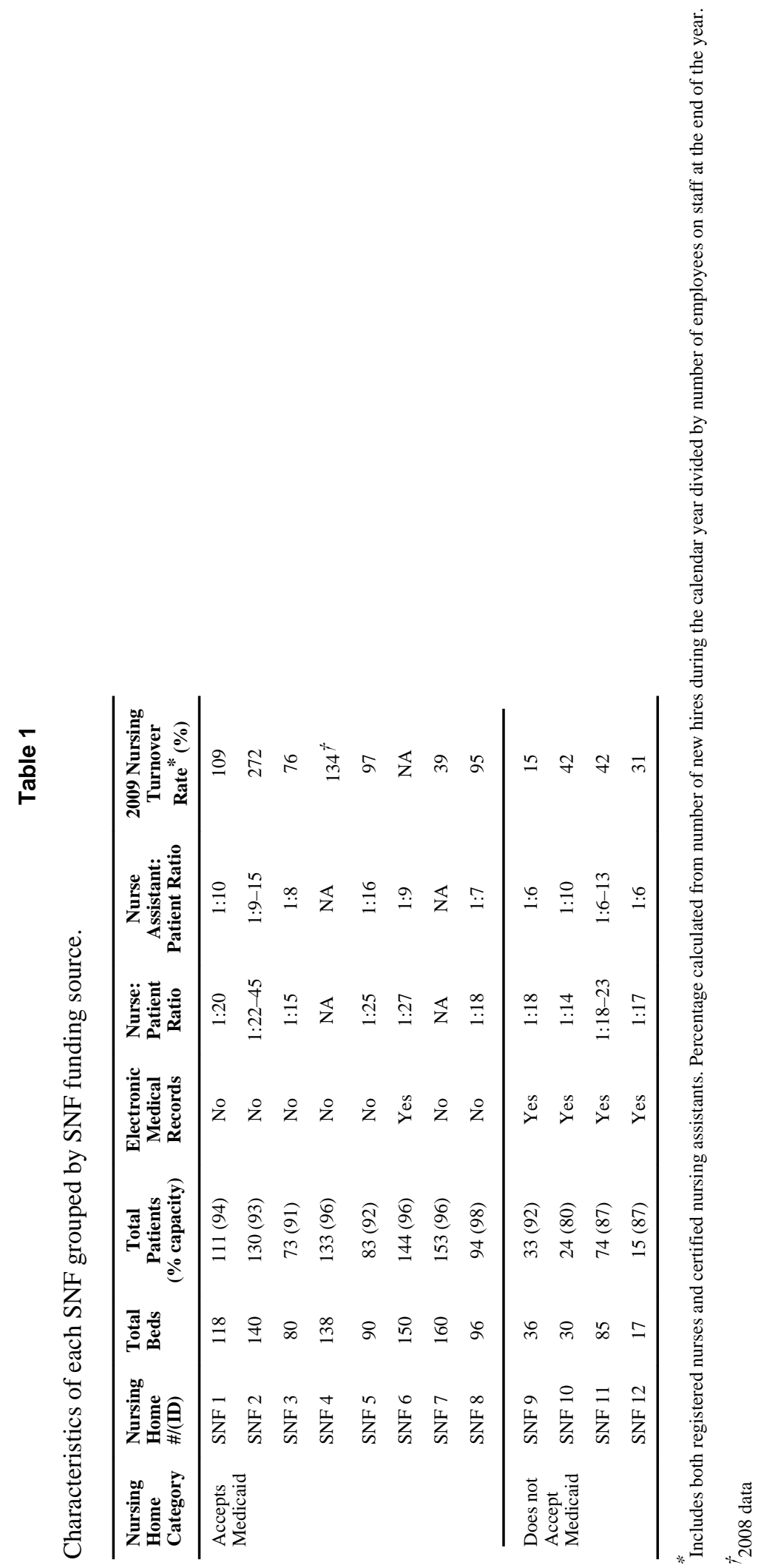


Table 2

Number $(\%)$ of patients with information available during the ED evaluation, grouped by SNF type.

\begin{tabular}{lcc}
\hline Information Item & $\begin{array}{c}\text { Medicaid SNFs } \\
(\mathbf{n = 9 5})\end{array}$ & $\begin{array}{c}\text { Private SNFs } \\
(\mathbf{n}=\mathbf{3 3})\end{array}$ \\
\hline Reason for visit & $78(81)$ & $31(94)$ \\
Past Medical History & $90(95)$ & $33(100)$ \\
Medications & $82(86)$ & $33(100)$ \\
Allergies & $89(94)$ & $33(100)$ \\
Usual Mental Status & $70(74)$ & $28(85)$ \\
Usual Ambulatory Status & $62(65)$ & $26(79)$ \\
Primary Provider Name & $74(78)$ & $26(79)$ \\
HCPOA ${ }^{\dagger}$ Name & $65(68)$ & $28(85)$ \\
Advance Directives ${ }^{\dagger}+$ & $67(71)$ & $31(94)$ \\
\hline
\end{tabular}

'Medicaid SNFs' refers to SNFs which do accept Medicaid; 'Private SNFs' refers to SNFs which do not accept Medicaid. p $<.05$

${ }^{\dagger}$ Health Care Power of Attorney

$t_{\mathrm{p}}^{+}<.01$ 
